\title{
Computing a Language-Based Guarantee for Timing Properties of Cyber-Physical Systems
}

\author{
Neil Dhruva \\ Birla Institute of Technology and Science, Pilani, India
}

Pratyush Kumar, Georgia Giannopoulou, Lothar Thiele
Computer Engineering and Networks Laboratory, ETH Zurich

\begin{abstract}
Real-time systems are often guaranteed in terms of schedulability, which verifies whether or not all jobs meet their deadlines. However, such a guarantee can be insufficient in certain applications. In this paper, we propose a method to compute a language-based guarantee which provides a more detailed description of the deadline miss patterns of an observed task. The only requirement of our method is that the timing behavior of the real-time system be modelled by a network of timed automata. We compute the language-based guarantee by constructing an equivalent finite state automaton in an iterative manner, using a counter-example guided procedure. We illustrate the language-based guarantee for two applications: design of a networked control system and scheduling in a mixed criticality system. In both cases, we show that the language-based guarantee leads to a more efficient design than the schedulability guarantee.
\end{abstract}

\section{INTRODUCTION}

Cyber-Physical Systems (CPS) interconnect computational and physical sub-systems which are often designed and analyzed under different theoretical foundations. Consider the example of a networked control system [1]. The timing properties of the communication network may be analyzed with real-time schedulability tests which guarantee whether all jobs meet their deadlines. On the other hand, given the worst-case sensor-to-actuator delay, the physical plant can be stabilized with a controller design for time delayed feedback systems [2]. A key challenge in the composition of such sub-systems is the provision of tight and compact guarantees.

The real-time systems community has primarily focused on providing schedulability as a guarantee. From the perspective of the real-time system, if a task-set is schedulable, i.e., if all jobs are guaranteed to finish before their deadlines, then and only then, will all other sub-systems perform correctly. A large body of research has investigated methods and tools to verify such a guarantee under different assumptions of task models, scheduling policies and resource considerations.

However, schedulability can be insufficient in certain settings. For the networked control system example, the physical plant may be able to withstand a few missed deadlines. Indeed, in [3], the authors show that a well-defined class of deadline hit and miss patterns can guarantee stability of a physical plant. Hence, it is pertinent to look for richer (more detailed) guarantees that extend beyond schedulability.

Researchers have proposed alternate richer guarantees. In [4], the authors proposed two metrics on the deadline hit and miss patterns, which they called $\mu$-patterns. A $\left(\begin{array}{l}n \\ m\end{array}\right) \mu$-pattern has at least $n$ deadline hits within $m$ consecutive jobs, and a $\left\langle\begin{array}{l}n \\ m\end{array}\right\rangle \mu$-pattern has at least $n$ consecutive deadline hits within

978-3-9815370-2-4/DATE14/C)2014 EDAA any $m$ consecutive jobs. These patterns can be generalized by the notion of regular languages as proposed in [3]. A slightly different approach was followed in [5]. The authors propose a dual guarantee. A nominal guarantee is the typical schedulability test, while an exceptional guarantee specifies for how long deadlines can be missed after an exceptional event.

For the above, a major challenge is the computation or verification of the guarantees. The $\mu$-patterns approach has been demonstrated for fixed-priority scheduling in [4]. The use of regular languages has been shown for time-triggered architectures in [6]. In [5], the settling-time approach is applied when modeling in Real-Time Calculus [7]. It is not clear how to compute these guarantees in a general setting.

Like in [3], we use a regular language that models the patterns of deadlines hits and misses as a guarantee. However, we focus on computing the guarantee for a broad class of scheduling algorithms and task models. To this end, we first represent the real-time system by a network of timed automata [8] in the model-checking tool UPPAAL [9]. Then, we aim to compute a language-based guarantee by model-checking for different candidate languages until we find the one that correctly represents the observed hit and miss patterns. Thus, our approach is limited only by the ability to model a realtime system as a network of timed automata and by the computational cost of model-checking.

In spite of their generic applicability, a common limitation of model-checking tools is the state-space explosion and the consequent high computation cost. Being conscious of this, we make two specific choices in our model-checking process. First, we use a structured approach to identify candidate languages to model-check. To this end, we employ an iterative procedure to incrementally compute a language of a given complexity that models the observed deadline hit and miss patterns. This is similar to the counterexample guided abstraction refinement (CEGAR) [10] approach proposed for program verification. Second, we gradually increase the complexity of the language we wish to use as the guarantee, while fully reusing the information gathered from earlier steps. Here, we define an uncertainty metric to decide when to terminate the iterative process of increasing the language complexity. With these two steps we propose a standard approach to compute the language-based guarantee.

We present two different applications of the language-based guarantee. First, using language inclusion, we demonstrate whether the guaranteed real-time performance matches the assumed performance by another sub-system. As an example, we choose the controller performance of an inverted pendulum with a Linear Quadratic Regulator. For chosen system parameters, the controller performance cannot be ascertained by the schedulability guarantee, but is ascertained by the languagebased guarantee. In the second application, we guide the design 
of mixed-criticality systems [11] to 'fairly' distribute resources among low-criticality tasks in exceptional cases when highcriticality tasks require more resources than usual. We do this using a worst-case deadline miss rate metric. Such a design step is usually not supported by schedulability analysis.

The rest of the paper is organized as follows. In Section II we discuss the model of the real-time system and formally define the guarantee we propose to compute. In Sections III and IV we formally describe the approach to compute the language-based guarantee. In Section $\mathrm{V}$ we present two applications of the language-based guarantee and illustrate them with numerical examples.

\section{SySTEM MODEL}

In this section, we will describe the model of the realtime system, define the language-based guarantee we want to compute, and illustrate these with an example.

\section{A. Real-Time System}

We consider a real-time system with a task-set, $T=$ $\left\{\tau_{0}, \tau_{1}, \ldots, \tau_{n}\right\}$, running on a single or multiple processors with blocking access to shared resources. We compute the languagebased guarantee (which characterizes the deadline hit and miss patterns of jobs) of a specific task, say $\tau_{i}$.

The only requirement of our approach is that the timing behavior of such a system be modeled by a network of timed automata [8], which we denote as $S$ (for system). Thus, our approach is restricted only by the modeling power of timed automata and the computational cost of model-checking.

\section{B. Guarantee Language $L_{G}$}

All observed deadline hit and miss patterns of the jobs of a task $\tau$ can be thought of as strings in a language, denoted as $L_{S}$ (for the system language). $L_{S}$ is over the alphabet $\Sigma=\{H, M\}$, where $H$ implies a hit event and $M$ implies a miss event. An example string is $(M H H)^{*}$, which is a periodic pattern with a deadline miss followed by two deadline hits.

We aim to identify a guarantee that closely, yet conservatively, approximates $L_{S}$. To this end, we compute a regular language [3], denoted $L_{G}$ (for the guarantee language), over the alphabet $\Sigma=\{H, M\}$. As we will see later, a regular language admits advantages which will be useful in the modelchecking. We say $L_{G}$ is a correct guarantee if

$$
L_{S} \subseteq L_{G}
$$

If a property is satisfied for all strings (or no string) of a correct guarantee $L_{G}$, then it follows that it is (or is not) satisfied on $L_{S}$. An example of such a property is: "Every deadline miss is followed by at least two deadline hits".

A regular language can be represented by an equivalent Deterministic Finite Automaton (DFA). In a DFA, every state encodes a finite observed history. Let $k$ denote the length of the longest history encoded by any state of a DFA, then we represent the DFA as $A_{k}$. The parameter $k$ controls the complexity of the corresponding language: a larger $k$ corresponds to a larger automaton and possibly a more accurate guarantee.

For the $A_{k}$ accepting our guarantee language, denoted $L_{G}(k)$, the history encoded in the states is the pattern of deadline hits and misses of the last $k$ jobs of task $\tau$. Then,
TABLE I. TASK MODEL FOR EXAMPLE 1

\begin{tabular}{ccccc}
\hline Task & Read & Execute & Write & Period \\
\hline$\tau_{0}$ & $1-2$ & $1-3$ & $1-2$ & 15 \\
$\tau_{1}$ & $1-2$ & $10-14$ & $1-2$ & 40 \\
$\tau_{2}$ & $1-3$ & $12-15$ & $1-3$ & 50 \\
\hline
\end{tabular}

TABLE II. GuARANTEE LANGUAGE $L_{G}(2)$ FOR EXAMPLE 1

\begin{tabular}{ccccc}
\hline Last Two Events & HH & HM & MH & MM \\
\hline Next Event & H or M & H or M & H or M & H \\
\hline
\end{tabular}

given a hit and miss pattern of the last $k$ jobs, $A_{k}$ can be used to determine if such a pattern can be observed in $L_{S}$, and if so, whether the next job would meet or miss its deadline. This is a Markov interpretation, where only the last $k$ hits and misses can influence the next job's deadline hit or miss. Similar ideas have been proposed in the analysis of cache hits and branch predictions [12].

Example 1: Consider a task-set $T$ with three periodic tasks $\tau_{0}, \tau_{1}, \tau_{2} \in T$ running on three separate cores and accessing a shared memory. Each task has three distinct phases: read data from memory, execute on respective cores using the read data, and write modified data back to memory. Such phased models of tasks have been shown to model many practical applications in the control and real-time domain [13]. The period and the minimum and maximum time units for each phase of each task (as computed, e.g. with static analysis,) are shown in Table I. Contention time before read or write is not included. The arbitration on the memory follows a nonpreemptive first-come-first-serve policy.

We examine $\tau_{0}$ for the deadline of $D_{0}=10$. Contention and blocking accesses to shared memory lead to variable response times of jobs of $\tau_{0}$ resulting in the deadline hit and miss patterns. Using the technique described in the next sections, we derive the language-based guarantee shown in Table II. This language corresponds to a DFA $A_{k}$ with $k=2$, i.e., given the deadline hit and miss pattern of the last two jobs, the language determines the guarantee for the next job. For instance, if $\tau_{0}$ encounters a deadline hit followed by a miss, i.e. $H M$, then the next event can be either a hit or miss. But, for two consecutive misses, i.e. $M M$, the next event is guaranteed to be a hit.

\section{Constructing $A_{k}$ FOR A Given $k$}

In this section, we will describe how we construct the DFA $A_{k}$ for a given value of $k$, such that the equivalent regular language $L_{G}(k)$ conservatively approximates $L_{S}$. We will first describe a specific template for $A_{k}$ which can be iteratively modified. We will then present an observer timed automaton which models our current estimate of $A_{k}$, denoted as $A_{k}^{i}$ for the $i^{\text {th }}$ iteration. We then verify a property to check if the estimated guarantee language conservatively approximates $L_{S}$. If a counterexample is generated, we show how to modify the observer, or equivalently construct $A_{k}^{i+1}$ from $A_{k}^{i}$. Finally, when no more counterexamples are generated and the iterative procedure terminates, we obtain $A_{k}$ and show properties of the corresponding $L_{G}(k)$.

\section{A. The Observer Automaton}

We begin by defining a template for $A_{k}$. The DFA $A_{k}$ is given by a 5-tuple $\left(Q, \Sigma, \delta, q_{0}, F\right)$, where $Q$ is the set of 
states, $\Sigma=\{H, M\}$ is the alphabet of accepted inputs, $\delta$ is the transition function defined as $Q \times \Sigma \rightarrow Q, q_{0} \in Q$ is the start state, and $F$ is the set of accepting states. The following are some properties of $A_{k}$.

- $\quad$ Each state $q \in Q$ corresponds to a particular string of size up to $k$ over the alphabet $\Sigma$. Thus, there are up to $2^{k+1}-1$ states.

- We can represent a state $q$ equivalently by its corresponding string denoted $s(q)$. For example, when the DFA is in a state $q$ with $s(q)=(M H H)$, the previous two jobs have met their deadlines while the one before them missed its deadline. The oldest information is the left-most. For the initial state $q_{0}$, we have $s\left(q_{0}\right)=\phi$.

- $\delta$ is a partial function, i.e., $\delta(q, x)$ does not have to be defined for every state $q \in Q$ and for both $x \in$ $\Sigma$. If the DFA is in state $q$ and the next input is $x$ where $\delta(q, x)$ is not defined, then the input string is not accepted by $A_{k}$, and thus does not belong to $L_{G}(k)$.

- The transition function follows from the string representation of the states. For example, let $q_{i}, q_{j}$ be two states with $s\left(q_{i}\right)=(M H H)$ and $s\left(q_{j}\right)=(H H M)$. If $\delta\left(q_{i}, M\right)$ is defined, then it is equal to $q_{j}$ for $k=3$.

- $F=\{q \in Q:|s(q)|=k\}$.

Thus, the template for $A_{k}$ defines a class of automata that accept any regular language with strings of length at least $k$. Any language, which can be represented as shown in Table II, is accepted by a DFA belonging to this class depending on the transitions defined in the DFA.

Example 2: For the language in Table II, the corresponding $A_{k}$ with the above template is shown in Fig. 1b.

For the template of $A_{k}$, we design a specific observer, denoted as $O$, as a timed automaton [8] in UPPAAL [9], such that:

- $\quad$ Each state of $A_{k}$ corresponds to a location of $O$.

- All possible transitions in $A_{k}$ are modeled in $O$. Every location in $O$ has two outgoing transitions for the two cases of hit and miss events. Transitions not defined in $A_{k}$ have a variable update $e=0$ (for enable) in $O$, while the others have a variable update $e=1$.

- $\quad$ Each transition in $O$ which corresponds to a transition in $A_{k}$ that accepts an $H$ (similarly $M$ ) has a synchronization channel receive-event hit? (miss?).

The network of timed automata, $S$, writes to the synchronization channel using send-events hit! and miss! whenever a job of task $\tau$ either meets or misses its deadline, respectively. Thus, for a particular execution trace, as the jobs of $\tau$ meet or miss deadlines, $S$ and $O$ synchronize over corresponding channels. We initialize $O$ such that the variable update rule on each transition is $e=0$. This corresponds to $A_{k}^{0}$ which has no defined transitions.

\section{B. Iterative Construction}

$S$ is extended to include the observer $O$. Then, (for the extended network of TA,) we verify the following (TCTL) safety property:

$$
\forall \square e=1 \text { (or equivalently, } A[] e==1 \text { in UPPAAL). }
$$

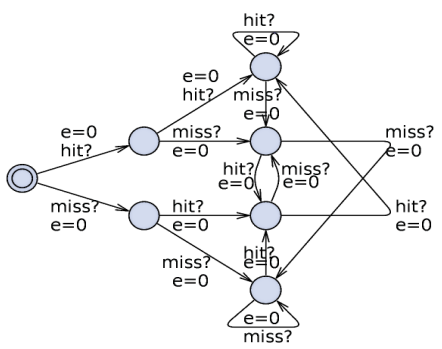

(a) Initial Observer $O$ for $k=2$.

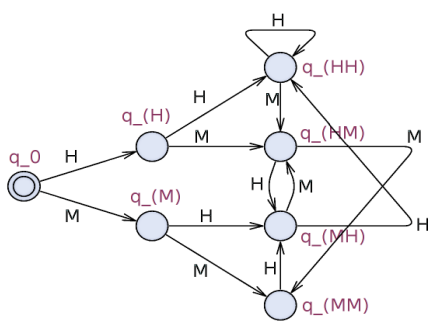

(b) Guarantee Automaton $A_{2}$.
Fig. 1. Refinement Illustration.

This property asserts that the variable $e$ equals 1 in all states. In terms of our usage of $e$, the property asserts that at all times the transitions taken by the observer are already defined in $A_{k}^{i}$. If the property is not satisfied, the modelchecker returns a counterexample which is a specific trace of inputs observed in the real-time system but not modeled by the observer. Then, we modify the observer such that the counterexample will not be generated again. The modification is direct: we set $e=1$ on the transition of $O$ which triggered the counterexample. Equivalently, we define (add) a new transition in $A_{k}^{i}$ to generate $A_{k}^{i+1}$. We then repeat the process of verifying property (2) with the modified observer.

\section{Properties of the Computed $L_{G}(k)$}

The iterative process will terminate after a finite number of steps, as in each step we define a new transition amongst the finite number of possible transitions of $A_{k}$. Upon termination, the states in DFA $A_{k}$ with no incoming transitions are dropped, and $A_{k}$ and the corresponding $L_{G}(k)$ satisfy the following:

Lemma 1 (Correctness): The language $L_{G}(k)$ corresponding to the DFA $A_{k}$ satisfies $L_{S} \subseteq L_{G}(k)$.

Lemma 2 (Tightness): If $A_{k}^{\prime}$ is obtained by disabling any single transition from $A_{k}$, then the corresponding language $L_{G}^{\prime}(k)$ does not satisfy $L_{S} \subseteq L_{G}^{\prime}(k)$.

The correctness property follows from the fact that asserting property (2) asserts $L_{S} \subseteq L_{G}(k)$. The tightness follows from the initialization wherein no transition of $A_{k}^{0}$ is defined. Thus, any defined transition in $A_{k}$ is due to an observed pattern of hits and misses in $S$.

Example 3: Consider the setup from Example 1. For $k=$ 2, we illustrate the observer in Fig. 1a. The guarantee automaton, shown in Fig. 1b, is then generated using the above procedure. The states are represented as $q \_y$, with $y=0$ for the start state and otherwise equal to the history corresponding to the state. The transition $M$ self-loop on $q_{-}(M M)$ is undefined at the end of the procedure and is thus removed. The computed $L_{G}(k)$ is shown in Table II.

\section{Guarantee Automaton For UnKnOwn $k$}

The previous section described how to compute $L_{G}(k)$ for a known parameter $k$. However, for applications in which it is unclear what $k$ should be, we need a method to choose the right $k$. This choice should balance between complexity and accuracy: for a large $k, L_{G}(k)$ can more accurately represent $L_{S}$, but can be computationally expensive to model-check. In this section, we present an iterative method that sequentially computes $L_{G}(1), L_{G}(2), \ldots$. We show how to fully utilize 


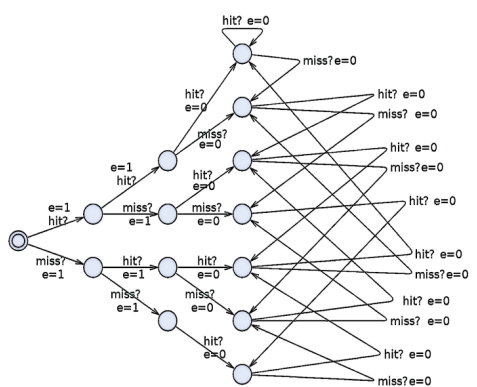

(a) Initial Observer for $k=3$ from $A_{2}$.

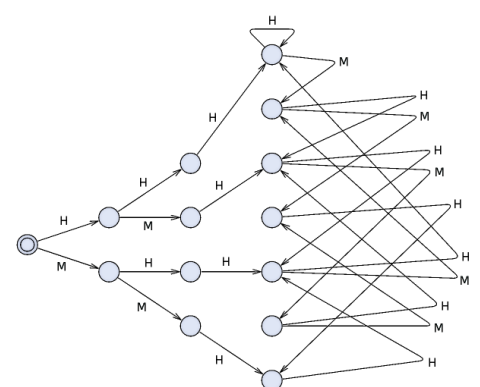

(b) Guarantee Automaton $A_{3}$.
Fig. 2. Refinement Illustration for Unknown $k$.

$L_{G}(k)$ when computing $L_{G}(k+1)$, propose a terminating condition for this method, and address scalability issues.

The following lemma forms the basis of our approach. It states that the computed language (as in the previous section) for a smaller $k$ cannot be a tighter approximation of $L_{S}$.

Lemma 3 (Inclusion): $L_{G}(k+1) \subseteq L_{G}(k), \forall k>0$.

We will argue the above result in terms of $A_{k}$ and $A_{k+1}$. For every state $q$ in $A_{k+1}$ we define a parent state $p(q)$ in $A_{k}$. Two cases arise based on the string representation $s(q)$. If $|s(q)|<k+1$, then $s(p(q))=s(q)$. If $|s(q)|=k+1$, then $s(p(q))$ is obtained by dropping the left-most (oldest) element in $s(q)$. This definition is such that, when processing a common input, if $A_{k+1}$ is in state $q$, then $A_{k}$ has to be in state $p(q)$. From the tightness of $A_{k+1}$ and the correctness of $A_{k}$, we have: if either or both transition(s) (out of $H$ and $M)$ are disabled in $A_{k}$ for state $p(q)$, then the corresponding transitions are also disabled in $A_{k+1}$ for state $q$. Thus, we cannot have a string that is accepted by $A_{k+1}$ and not by $A_{k}$.

\section{A. Refining $L_{G}(k)$ by Increasing $k$}

We provide an iterative refinement approach for increasing $k$ till we get a satisfactory $L_{G}(k)$. We start with the observer for $k=1$, modify it using the procedure in the previous section, and derive $A_{1}$. Then, we begin the process for $A_{2}$. However, we can now use information from $A_{1}$ to modify $A_{2}^{0}$ before iterative verification with the model-checker. We know that for every state $q$ in $A_{2}^{0}$, if the parent state $p(q)$ in $A_{1}$ has either or both transition(s) disabled, then the corresponding transitions will also be disabled in $A_{2}$. Using this we modify $O$, and hence $A_{2}^{0}$, by removing certain transitions. The modified observer is now used to obtain $A_{2}$. This process of constructing $A_{k+1}$ by using information from $A_{k}$ is termed refinement.

Example 4: Consider guarantee automaton $A_{2}$ shown in Fig. 1b. Using this we initialize the observer for $k=3$, shown in Fig. 2a. Note that certain transitions are removed from the observer. For instance, the state $q$ with $s(q)=(H M M)$ does not have a $\delta(q, M)$ transition. Also, state $q$ with $s(q)=$ $(M M M)$ is not reachable at all and is thus removed. With this observer and the procedure of the previous section, we compute the guarantee automaton $A_{3}$ shown in Fig. 2 b.

This process is complete in the sense that any counterexample generated when computing $A_{k}$ will not be generated when computing $A_{k+1}$. This reduces the model-checking time.
TABLE III. UNCERTAINTY METRIC FOR EXAMPLE 1

\begin{tabular}{ccccccccc}
\hline$k$ & 1 & 2 & 3 & 4 & 5 & 6 & 7 & 8 \\
\hline$U(k)$ & 1.000 & 0.929 & 0.733 & 0.500 & 0.317 & 0.197 & 0.120 & 0.072 \\
Iterative & 0.949 & 2.219 & 4.335 & 6.798 & 9.695 & 13.901 & 19.401 & 27.635 \\
Non-iterative & 0.948 & 2.276 & 4.750 & 7.800 & 11.824 & 18.438 & 29.786 & 53.339 \\
\hline
\end{tabular}

\section{B. Terminating Condition}

We propose Uncertainty Metric, denoted $U(k)$, to define a terminating condition for the iterative process of increasing $k$

$$
U(k)=\frac{e_{p r}}{e_{p o}},
$$

where $e_{p r}$ is the number of transitions present in $A_{k}$, and $e_{p o}=2^{k+2}$, the total number of transitions possible in $A_{k}$. The metric conveys the level of uncertainty in $A_{k}$ with respect to the predictions at each state, and is similar to the statistical metric of entropy.

$U(k)$ varies between 0 and $1 . U(k)=1$ implies that every state in $A_{k}$ has two outgoing transitions. Thus, irrespective of the previous sequence of hits and misses, the next event can either be a hit or miss. A lower value of $U(k)$ implies that there is lesser uncertainty, because there are states with either one or no outgoing transitions.

From Lemma 3 , it is clear that $A_{k+1}$ will have a maximum of twice the number of transitions as $A_{k}$. $e_{p o}$ increases to twice the value for each increase in $k$. Hence, the ratio either remains constant or decreases with an increase in $k$, i.e.,

Lemma 4: $U(k+1) \leq U(k)$.

Using the above property, we can decide to terminate the iterative process if $U(k)$ reaches a value close to 0 . However, for some settings, uncertainty may be inevitable. For instance, if every job of $\tau$ can either hit or miss its deadline, then $U(k)=1$ irrespective of $k$. Thus, in addition to the absolute terminating condition, we also have a relative condition, such as $U(k+1) / U(k)$ is a value close to 1 .

Example 5: For the running example, the uncertainty metric for different values of $k$ is shown in Table III. Row 3 shows time (in s) required to compute each $A_{k}$ using the iterative refinement approach (Subsection IV-A). Row 4 shows time (in s) required if each $A_{k}$ is computed non-iteratively, i.e., without using the iterative refinement procedure. These readings are computed on an Intel ${ }^{\circledR}$ Core $^{\mathrm{TM}} 2$ Quad CPU Q6600 @ $2.40 \mathrm{GHz} \times 4$ machine with $4 \mathrm{GiB}$ RAM. With increase in $k$, the iterative approach shows a significant performance gain over the non-iterative approach.

\section{Scalability}

For different representative examples, we computed the guarantees for $k$ from 1 to 4 . For FCFS and fixed priority arbitration on the bus, systems with up to five cores could be analyzed within 18 hours on a server-grade Intel ${ }^{\circledR} \mathrm{Xeon}^{\circledR}$ @ 2.90Ghz. With TDMA arbitration, each core can be analyzed in isolation. Details of the experiments are in the thesis [14].

\section{Applications}

In this section, we will present applications of the language-based guarantee. First, we will show how to verify a controller's performance requirement by a language inclusion 
test. Second, we will show how to compare scheduling algorithms for mixed-criticality systems by computing worst-case deadline miss rates for low-criticality tasks.

\section{A. Language Inclusion}

In model-based design, a standard step is to check that all assumptions are satisfied by the guarantee. Let $L_{A}$ denote the language of hit and miss patterns which meet given performance constraints. Then, the real-time system $S$ satisfies these constraints if $L_{S} \subseteq L_{A}$. It is sufficient to show that $L_{G} \subseteq L_{A}$, under the correctness property (Lemma 1).

We illustrate language inclusion for the design of a networked control system [1]. Consider an inverted pendulum on a moving cart. Let mass of the cart be $0.5 \mathrm{~kg}$, the mass, inertia and length of the pendulum be $0.2 \mathrm{~kg}, 0.006 \mathrm{~kg} . \mathrm{m}^{2}$ and $0.3 \mathrm{~m}$, respectively. Let the coefficient of friction of the cart be $0.1 \mathrm{~N} / \mathrm{m} / \mathrm{s}$. The state of this control plant is given by

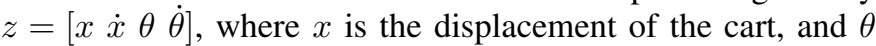
the angular displacement of the pendulum, and derivatives are denoted with a dot on top. With period $P=0.6 \mathrm{~s}$, we sense the state and compute a feedback control to change $\dot{x}$ and $\dot{\theta}$.

Assuming a sensor-to-actuator delay of exactly one period, we design a Linear Quadratic Regulator (LQR) [15]. Whenever the delay is larger than a period, we do not actuate the controller output and the plant is in an open-loop configuration. Thus, we have a switched linear time-invariant system. We can collate the plant and controller state variables at the $n^{\text {th }}$ sampling time by $X[n]$ with the following dynamics

$$
\begin{aligned}
& X[n+1]=A_{c l} X[n], \quad \text { if } d[n] \leq P, \\
& X[n+1]=A_{o l} X[n], \quad \text { otherwise, }
\end{aligned}
$$

where $A_{c l}$ and $A_{o l}$ are the system matrices for closed-loop and open-loop, respectively, $d[n]$ is the sensor-to-actuator delay for the $n^{\text {th }}$ period, and $P$ is the sampling period. For the computed LQR controller, we have $\left\|A_{c l}\right\|=0.82$, and $\left\|A_{o l}\right\|=28.2$.

Let the given performance requirement be: the augmented state variable $X$ must be exponentially stable such that

$$
\frac{\|X[n+6]\|}{\|X[n]\|}<0.5, \quad \forall n>0, X[n] .
$$

This condition specifies that the energy in the vector $X$ must at least halve in every 6 periods. This is a stronger condition than asymptotic stability. This condition may be satisfied for different deadline hit and miss patterns of 6 consecutive control signals. Let a such a pattern by represented as $\sigma=\left(\sigma_{1}, \ldots, \sigma_{6}\right)$, where $\sigma_{i} \in\{H, M\}$. For a given pattern, there is a corresponding matrix $A_{\sigma}=\prod_{i=1, \ldots, 6} A_{i}$, where $A_{i}=A_{c l}$ if $\sigma_{i}=H$ and $A_{i}=A_{o l}$ if $\sigma_{i}=M$. As shown in [3], condition of (4) is satisfied for a pattern $\sigma$ if the corresponding matrix $A_{\sigma}$ has an eigenvalue less than 0.5 . All patterns satisfying this condition are specified as a regular language $L_{A}$. The minimized automaton corresponding to $L_{A}$ for the computed matrices $A_{c l}$ and $A_{o l}$ is shown in Fig. 3a.

The controller is implemented in a distributed real-time system: (a) sensed data is sent via a shared bus to the controller, (b) control output is computed on a dedicated processing unit, (c) control outputs are sent via the same shared bus back to the plant. There are two other tasks which also read data via the bus, compute and write data back through the bus. The

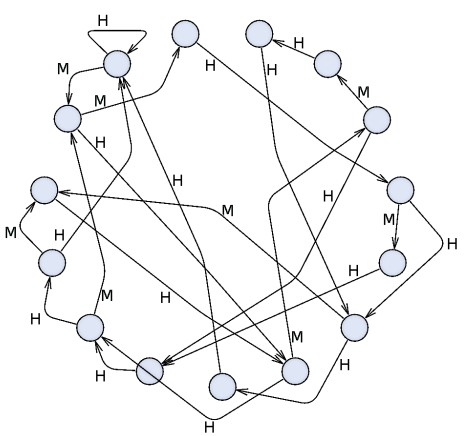

(a) Minimized Automaton for $L_{A}$.

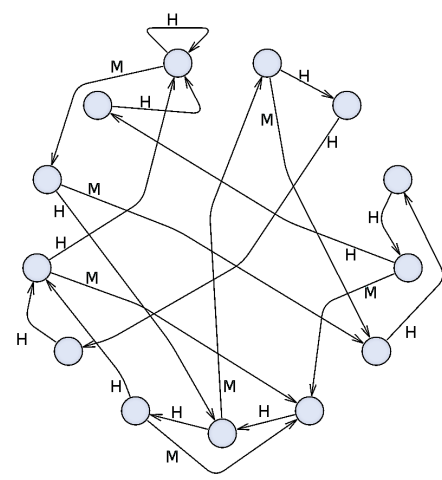

(b) Minimized Automaton for $L_{G}$.
Fig. 3. The Minimized Automata.

TABLE IV. TASK PARAMETERS FOR THE CONTROL EXAMPLE

\begin{tabular}{ccccc}
\hline Task & Read Range & Exec Range & Write Range & Period \\
\hline$\tau_{0}$ & $0.06-0.18$ & $0.06-0.12$ & $0.06-0.12$ & 0.6 \\
$\tau_{1}$ & $0.9-1.02$ & $0.06-0.12$ & $0.06-0.12$ & 2.4 \\
$\tau_{2}$ & $1.38-1.5$ & $0.06-0.12$ & $0.06-0.18$ & 3.6 \\
\hline
\end{tabular}

arbitration policy on the bus is non-preemptive first-come-firstserve. Thus, the bus is a shared resource which can increase the sensor-to-actuator delay. The minimum and maximum time (in s), for each phase of each task is shown in Table IV. Contention time before read or write is not included. $\tau_{0}$ is the controller task for the inverted pendulum with a period of $0.6 \mathrm{~s}$.

Applying the method of [16], the worst-case response time of $\tau_{0}$ is $0.72 \mathrm{~s}>P$. Using the schedulability guarantee, the plant can never be guaranteed to be in the closed-loop mode, and thus is not guaranteed to meet the requirement of (4). Further, no amount of speed-up of the processor executing $\tau_{0}$ can meet the controller requirement. One would have to speed up the bus by at least $37.5 \%$ to satisfy the requirement.

Since the given $L_{A}$ accepts strings of size 6 , we compute $L_{G}$ for $k=5$. Fig. $3 \mathrm{~b}$ shows the corresponding minimized automaton. We then compute $L_{\text {diff }}=L_{G} \cap \neg L_{A}$, where $\neg L_{A}$ is the language complement. We verified that $L_{\text {diff }}=\phi$, and thus $L_{G} \subseteq L_{A}$. This shows that the real-time system can meet the controller constraint of (4), contrary to the conclusion from the schedulability guarantee. More details are in [14].

\section{B. Calculating Worst-Case Deadline Miss Rate}

While the language-based guarantee is very detailed, it can be used to compute specific metrics. An example of this is the worst-case deadline miss rate. Let $w$ be an infinite length string of deadline hits and misses, where the $i^{t h}$ element $w_{i} \in$ $\{H, M\}$. The worst-case deadline miss rate WMR is given as

$$
\operatorname{WMR}=\max _{w \in L_{S}} \frac{\left|\left\{i: w_{i}=M\right\}\right|}{|w|} .
$$

From the correctness property (Lemma 1), computing the WMR with $L_{G}$, instead of $L_{S}$, is a safe over-approximation.

Given $A_{k}$ corresponding to $L_{G}$, we compute WMR as follows:

- We construct a graph $G^{\prime}=\left(V^{\prime}, E^{\prime}, \rho\right)$ where the vertices $V^{\prime}$ correspond to the states in $A_{k}$, the edges $E^{\prime}$ are the defined transitions in $A_{k}$, and weights 
TABLE V. MIXED CRITICALITY TASK-SET

\begin{tabular}{ccccccc}
\hline$\tau_{i}$ & $\chi_{i}$ & Read & Write & Exec & Period & Deadline \\
\hline$\tau_{0}$ & HI & $5-7$ & $6-9$ & $7-8$ & 80 & 80 \\
$\tau_{1}$ & LO & $4-6$ & $7-8$ & $5-6$ & 50 & 30 \\
$\tau_{2}$ & LO & $3-5$ & $7-9$ & $6-7$ & 60 & 30 \\
\hline
\end{tabular}

TABLE VI. MIXED CRITICALITY RESUlTS

\begin{tabular}{ccccccc}
\hline & \multicolumn{2}{c}{$\mathrm{PA}_{1}$} & & \multicolumn{2}{c}{$\mathrm{PA}_{2}$} \\
\cline { 2 - 3 } \cline { 5 - 6 } & $\tau_{1}$ & $\tau_{2}$ & & $\tau_{1}$ & $\tau_{2}$ \\
\hline$k$ & 9 & 12 & & 15 & 8 \\
$W M R$ & 0.3125 & 0.75 & & 0.5415 & 0.25 \\
\hline
\end{tabular}

$\rho: E \rightarrow\{-1,1\}$ is -1 (similarly 1 ) on edges if the corresponding transition accepts $M(H)$.

- We compute the minimum cycle mean of $G^{\prime}$, denoted MCM, with the Minimum Cycle Mean algorithm [17].

- $\quad$ Then, $W M R=\frac{1-M C M}{2}$.

We use WMR to compare scheduling algorithms for mixedcriticality systems [11]. In mixed-criticality systems, every task $\tau_{i}$ has a defined criticality level say $\chi_{i}$, and at any given time, $t$, there is a global criticality level say $\chi_{g}(t)$. If $\chi_{g}(t)>\chi_{i}$ then most existing scheduling policies decide to drop task $\tau_{i}$ from $t$ onwards. In other words, when the global criticality level rises due to certain exceptional run-time events, low criticality tasks are no more scheduled. We believe this abrupt dropping of tasks is due to the limitation of the schedulability guarantee. Instead, we may still run the low criticality tasks under reduced performance guarantees, in particular with higher WMR.

Consider a dual-criticality (HI, LO) task-set with three tasks executing on independent processing cores, but interfering on a shared bus which follows a non-preemptive fixedpriority arbitration policy. Tasks read data via the bus, compute using the read data and write data back via the bus. Read and write is done through individual accesses, each requiring 1 time unit once access to the bus is granted. The ranges of read-write access requests and execution times (in time units) for the different tasks, when the global criticality level is HI, are shown in Table V. In this case, tasks $\tau_{1}$ and $\tau_{2}$ may not be guaranteed to meet all their deadlines. But we can guarantee a certain WMR for each.

We consider two priority assignments, $\mathrm{PA}_{1}=\tau_{0}>\tau_{1}>\tau_{2}$ and $\mathrm{PA}_{2}=\tau_{0}>\tau_{2}>\tau_{1}$. For both, we compute the languagebased guarantee for $\tau_{1}$ and $\tau_{2}$. We do not have a pre-defined $k$ so we use our iterative refinement procedure to increase $k$ with the terminating condition: $U(k)<0.1$ or $U_{k+1} / U_{k}>0.9$. We then compute WMR for each task of both priority assignments. The results in Table VI are interpreted as follows:

- Fairness amongst LO-criticality tasks: The absolute value of the difference between WMR of $\tau_{1}$ and $\tau_{2}$ is smaller for $\mathrm{PA}_{2}$.

- Responsiveness of LO-criticality tasks: The sum of the WMR of $\tau_{1}$ and $\tau_{2}$ is higher for $\mathrm{PA}_{2}$.

Hence, $\mathrm{PA}_{2}$ is clearly a better priority assignment. However, when using only the schedulability guarantee, the two priority assignments are indistinguishable in the HI-criticality mode, and both $\tau_{1}$ and $\tau_{2}$ will be dropped in either case. We can similarly compare two different scheduling policies using the language-based guarantee.

\section{CONCLUSION}

We proposed a language-based guarantee that details the deadline hit and miss patterns of the jobs of a task, then provided a method to compute this guarantee and finally presented two applications to describe its applicability. We also showed the superiority of the language-based guarantee against the schedulability guarantee. With a wide scope of application, the only limitation of our method is the inevitable cost of model-checking. Yet, we have reduced such costs by using a suitable observer template and corresponding modification procedure, and an iterative refinement approach that reuses information from one iteration to the next.

In conclusion, we believe the language-based guarantee is a viable option for the design and analysis of cyber-physical systems. We are interested to study the implications of the detailed guarantee in other application domains.

Acknowledgement: This work was partially funded by the European Union Seventh Framework Programme (FP7/20072013) under grant agreement number 288175.

\section{REFERENCES}

[1] A. Bemporad, M. Heemels, and M. Johansson, Networked control systems. Springer, 2010, vol. 406.

[2] J. P. Hespanha, P. Naghshtabrizi, and Y. Xu, "A survey of recent results in networked control systems," Proceedings of the IEEE, vol. 95, no. 1, pp. 138-162, 2007.

[3] R. Alur and G. Weiss, "Regular specifications of resource requirements for embedded control software," in RTAS, 2008, pp. 159-168.

[4] G. Bernat, A. Burns, and A. Llamosí, "Weakly hard real-time systems," IEEE Trans. Computers, vol. 50, no. 4, pp. 308-321, 2001.

[5] P. Kumar and L. Thiele, "Quantifying the effect of rare timing events with settling-time and overshoot," in RTSS, 2012, pp. 149-160.

[6] A. D'Innocenzo, G. Weiss, R. Alur, A. J. Isaksson, K. H. Johansson, and G. J. Pappas, "Scalable scheduling algorithms for wireless networked control systems," in CASE, 2009, pp. 409-414.

[7] L. Thiele, S. Chakraborty, and M. Naedele, "Real-time calculus for scheduling hard real-time systems," in ISCAS, 2000, pp. 101-104.

[8] R. Alur and D. L. Dill, "A theory of timed automata," Theoretical Computer Science, vol. 126, pp. 183-235, 1994.

[9] K. G. Larsen, P. Pettersson, and W. Yi, "Uppaal in a nutshell," International Journal on Software Tools for Technology Transfer (STTT), vol. 1, no. 1, pp. 134-152, 1997.

[10] E. Clarke, O. Grumberg, S. Jha, Y. Lu, and H. Veith, "Counterexampleguided abstraction refinement," in Computer aided verification. Springer, 2000, pp. 154-169.

[11] S. Baruah, H. Li, and L. Stougie, "Towards the design of certifiable mixed-criticality systems," in RTAS, 2010, pp. 13-22.

[12] I. Hur and C. Lin, "Adaptive history-based memory schedulers," in MICRO, 2004, pp. 343-354.

[13] R. Pellizzoni, E. Betti, S. Bak, G. Yao, J. Criswell, M. Caccamo, and R. Kegley, "A predictable execution model for cots-based embedded systems," in RTAS, 2011, pp. 269-279.

[14] N. Dhruva, "Crossing the Deadline: An Automata-Based Hard Real-Time Guarantee,” Bachelor's Thesis, ETH Zurich, 2013. [Online]. Available: ftp://ftp.tik.ee.ethz.ch/pub/students/2013-HS/BA2013-17.pdf

[15] J. Hespanha, "Lecture notes on lqr/lqg controller design." [Online]. Available: http://www.uz.zgora.pl/ wpaszke/materialy/kss/lqrnotes.pdf

[16] G. Giannopoulou, K. Lampka, N. Stoimenov, and L. Thiele, "Timed model checking with abstractions: Towards worst-case response time analysis in resource-sharing manycore systems," in EMSOFT, 2012, pp. 63-72.

[17] R. M. Karp, "A characterization of the minimum cycle mean in a digraph," Discrete Mathematics, vol. 23, no. 3, pp. 309 - 311, 1978. 